\title{
How integrated are cryptocurrencies
}

\section{Moinak Maiti*, Darko Vukovic, Viktor Krakovich and Maneesh Kumar Pandey}

\author{
Department for Finance, \\ St. Petersburg School of Economics and Management, \\ National Research University Higher School of Economics, \\ Kantemirovskaya St. 3A, Sankt Petersburg 194100, Russia \\ Email:mmaiti@hse.ru \\ Email: vdarko@hotmail.rs \\ Email: vkrakovich@hse.ru \\ Email: maneeshban@gmail.com \\ *Corresponding author
}

\begin{abstract}
The present study focuses on five cryptocurrencies co-movements physiognomies both in time and frequency domain. The present study highlighted several interesting facts related to cryptocurrencies co-movements both in time and frequency domain that have high policy and investment implications. Overall wavelet coherence diagrams clearly indicate about the very short and long contagion effect among the cryptocurrency pairs for the whole study period. The contagion effect is different at different time scales. Finally wavelet clustering diagram indicates that by investing only in XBP and BitCoin cryptocurrencies investors are not going to get any benefit from diversification. This predictable co-movements pattern among the cryptocurrencies could be the basic investment strategies to gain maximum profit by diversifying the risk in cryptocurrency investments.
\end{abstract}

Keywords: cryptocurrency; co-movement; XBP; BitCoin; wavelet; wavelet coherence; wavelet clustering; diversification; investment decision.

Reference to this paper should be made as follows: Maiti, M., Vukovic, D., Krakovich, V. and Pandey, M.K. (2020) 'How integrated are cryptocurrencies', Int. J. Big Data Management, Vol. 1, No. 1, pp.64-80.

Biographical notes: Moinak Maiti is an Associate Professor in the Department of Finance, and a Research Fellow of the International Laboratory of Intangible-Driven Economy, National Research University-Higher School of Economics, Saint Petersburg. He teaches EPAS and ACCA accredited Master courses in Finance and CIMA accredited Bachelor courses. He is an active speaker and invited to share the World renowned platforms of World Bank, United Nations, World Trade Organisation (WTO Studies), SAS ${ }^{\circledR}$, Bloomberg LP, Max Plank Society, PEP Canada, Ivey League Universities and many others. Presently, he is in the editorial board of several journals published by Emerald, Wiley and Elsevier.

Darko Vukovic is an Associate Professor at the National Research University Higher School of Economics, St. Petersburg School of Economics and Management, Department of Finance. He is a Research Fellow at the Geographical Institute 'Jovan Cvijic' of the Serbian Academy of Sciences and Arts and the Chief of Department of Regional Geography. His main topics of research are financial markets, portfolio management, investments, and 
regional economics. He is a member of many national and international professional bodies, also member of editorial board of several international journals. In 2016, he registered international patent in the field of agglomeration economy. In 2017, he received his Honorary Doctor of Excellence Diploma from the CIAC - Confederation of International Accreditation Commission, Canada, New Delhi in 2017 and from the KEISIE - International University (KIU), South Korea in 2017. In same year he received a certificate for outstanding excellence in Regional Economics from the Indian Management Academy.

Viktor Krakovich is Senior Lecturer in the Department of Finance, HSE SPB. $\mathrm{He}$ is associated with corporate finance, financial risk management and other courses of the department.

Maneesh Kumar Pandey has a wide experience in working with IT industries and works in different countries in Asia. Presently his focus is to research on financial market with application of analytics.

\section{Introduction}

Cryptocurrency is relatively new as compared to other financial products and it is fundamentally based on new blockchain technology. Though the blockchain technology is still beyond the scope of understanding of many investors but still just in a short period of half a decade time cryptocurrencies get special attention, since 2013 when BitCoins start trading actively. The reason could be cryptocurrencies functions similar like other traditional assets that are traded in the financial markets. Cryptocurrency phenomenon is relatively new and requires further comprehensive research to explore the hidden information. Since its inception the key issues that researches or investors try to answer is "whether the dynamic nature of cryptocurrencies can be predictable and whether it follows EMH (efficient market hypothesis)" as explained by Malkiel and Fama (1970). Liu and Tsyvinski (2018) studies with three cryptocurrencies namely BitCoin, Ripple and Ethereum find that there is a trade-off between these cryptocurrencies and stocks, currencies and precious metals. The study also confirms that cryptocurrencies are not affected by the common stock market and macroeconomic factors. Cryptocurrency are affected by certain factors that are quite specific to cryptocurrency market. That suggests that though cryptocurrency have few common behaviours like common stocks but it is not much affected or influenced by the common stock market factors. That clearly indicates cryptocurrencies need comprehensive study and explores the hidden properties of it.

Several studies in the past investigate about the co-movement of stock markets globally (see Granger and Morgenstern, 1970; Chaudhuri, 1997; Vyklyuk et al., 2013; Patel, 2017; Bhuiyan et al., 2017; many others). There are studies which show that the co-movements among the stock markets are both for shorter durations (see Forbes and Rigobon, 2002) and longer durations (see Markwat et al., 2009). Fundamentally if there exists a higher correlation between the two markets than investors are not going to get much benefitted from the portfolio diversifications. All these studies mentioned above are done to study the co-movements among the stock markets across the globe. If there is a 
co-movement among the cryptocurrencies then it will indicates that cryptocurrencies are separate asset classes that reacts to a particular stimulus in common. It will indicates that cryptocurrencies has special characteristics or behaviour that differs from other asset classes available in the market. Then in such case it will be important to identify such factors that influence cryptocurrencies price movement or determinations. Virtually no study has done to address the co-movements among the cryptocurrencies both in time and frequency domain together.

The present study addresses comprehensively about the co-movement among the five cryptocurrencies (BitCoin, Dash, Monero, Stellar and XBP) both in time and frequency domain. Moreover it identifies the pair of cryptocurrencies in which by including those in the investment portfolio one can achieve maximum diversification. The present study is novel for several reasons: First, virtually no study has done to address the co-movements among the cryptocurrencies both in time and frequency domain together. Second, it identifies the pair of cryptocurrencies in which by including those in the investment portfolio one can achieve maximum diversification. Third our study period and methodology used are completely different from the previous studies. This predictable co-movements pattern could be the basic investment strategies to gain maximum profit by diversifying the risk in cryptocurrency investments. The structure of the present work is as follows: it starts with introducing the past and present status of the cryptocurrencies that covers the introduction part of the paper. Literature review sections covers all the important studies related to the topic and discuss about the need of the present study in Section 2. Data and methodology in Section 3 cover comprehensibly about the data and the methodology used, and its appropriateness related to the present study. Then result and discussion in Section 4 comprehensively discusses about the findings in details and its implications. Finally study concluded by discussing the important conclusions of the study and its policy implications followed by the future directions of research in Section 5 .

\section{Review of literature}

Though the phenomenon of cryptocurrencies evolved in 2009 but starts active trading in 2013 and onwards. Thereafter, cryptocurrencies market increases exponentially since its inception and attracts major attentions due to its similar functioning like other traditional assets that are traded currently in the financial markets. Cryptocurrency phenomenon is relatively new and as result of which very limited number of studies are done on cryptocurrencies. The behaviour of cryptocurrency is very critical and not fully explained by any existing financial theories. In addition to it, the future of cryptocurrency is still a big question due to lack of protection mechanism from the regulatory body. European Central Bank (2012) indicates about the financial instability that can arise due to the increase in growth of cryptocurrencies and its integration with the global economy. Followed by European Banking Authority (2014) and Financial Action Task Force (2014) endorse that lack of specific regulatory protection regulations for cryptocurrencies may leads to high losses, business crashes and terrorist funding. Academically the issue of risk associated with cryptocurrencies from policy perspective were comprehensively highlighted by Vandezande (2017) study. 
Cryptocurrencies literature on price dynamics and speculation bubbles is highly uneven. Cheah and Fry (2015) concluded that cryptocurrencies are more prone to speculation bubbles whereas Blau (2017) argued that high volatility nature of cryptocurrency is not due to the high speculations. Other than Blau (2017) several other researcher like Katsiampa (2017), Pieters and Vivanco (2017), etc. study the volatility of cryptocurrencies. Differences in the findings among the research leads debate on whether cryptocurrency are currencies or like any other speculative investment instruments. Bariviera et al. (2017) added that cryptocurrency lacks all major properties of currency. To consider cryptocurrencies as group of separate asset class, it must provide enough evidence of similar patterns in terms of the co-integrations or co-movements among themselves in response to a common shock.

Kurihara and Fukushima (2017) and Caporale and Plastun (2017) study address anomalies in cryptocurrencies market. Then Caporale et al. (2018) study reflects on the persistence in the cryptocurrency market. Several researchers like Bouri et al. (2017a, 2017b), Corbet et al. (2018), and Ji et al. (2018) study the interlinkages between cryptocurrency and other asset classes. Bouri et al. (2017a) and Corbet et al. (2018) study uses GARCH. Recently, Ji et al. (2018) study find dynamic connectedness and integration among the six cryptocurrencies. The period of the study is from 7 August 2015 to 22 February 2018 which is comparatively of shorter duration. All of these previous studies address the interlinkages between the cryptocurrencies and other asset classes like VIX, gold and other indexes are analyses. None of them exclusively addressed whether there is co-movement among cryptocurrencies and if so then at what level. The present study addresses the above questions and it identifies the pair of cryptocurrencies by including them in the investment portfolio one can achieve maximum diversification. The present study addresses the following which are not addresses by previous studies: First, virtually no study has done to address the comovements among the cryptocurrencies both in time and frequency domain together. Second, it identifies the pair of cryptocurrencies in which by including those in the investment portfolio one can achieve maximum diversification. Third our study period and methodology used are completely different from the previous studies. This predictable co-movements pattern could be the basic investment strategies to gain maximum profit by diversifying the risk in cryptocurrency investments.

\section{Data and methodology}

The study focus on the five crypto currencies namely BitCoin, Dash, Monero, Stellar and XBP, are selected based on the highest market capitalisation and longer duration. The data are in daily frequency from 6th August 2014 to 28th September 2018 and all data samples are collected from CoinMarketCap (https://coinmarketcap.com/coins/). The present study uses wavelet techniques to derive the study conclusions. The study starts with plotting graphs obtained from the daily returns of the series. Then it decomposes the series at different scales using wavelets techniques to see any significant variations in the findings. Finally it plots clustering diagram to check the possible ways to get benefits from the investment diversifications. Details of it are discussed in the succeeding paragraphs. 
Figure 1 Cryptocurrencies time series multi scale decomposition plot for the daily returns, (a) Stellar (b) Dash (c) Monero (d) XBP (e) BitCoin

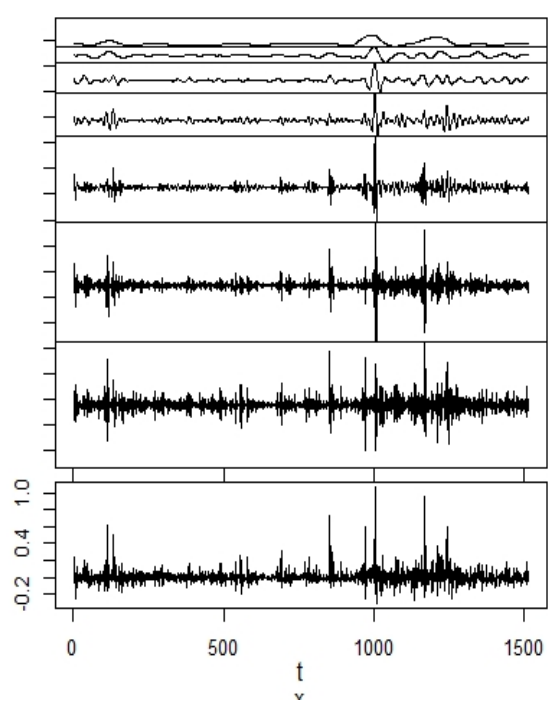

(a)

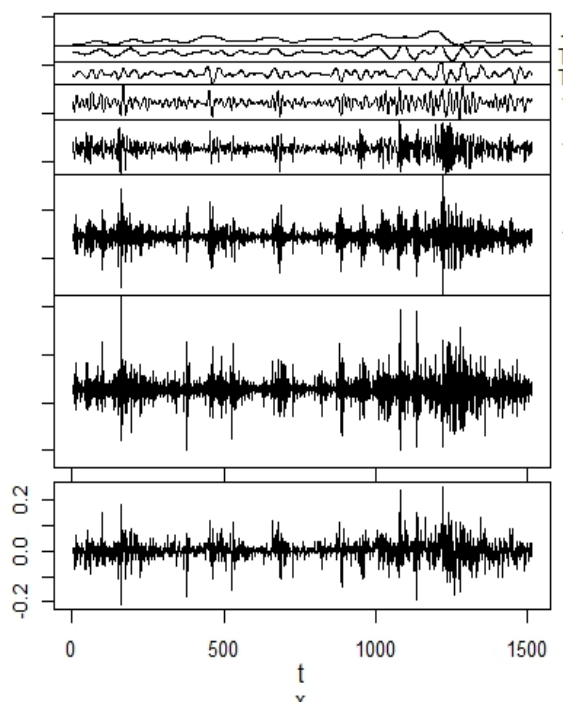

(c)
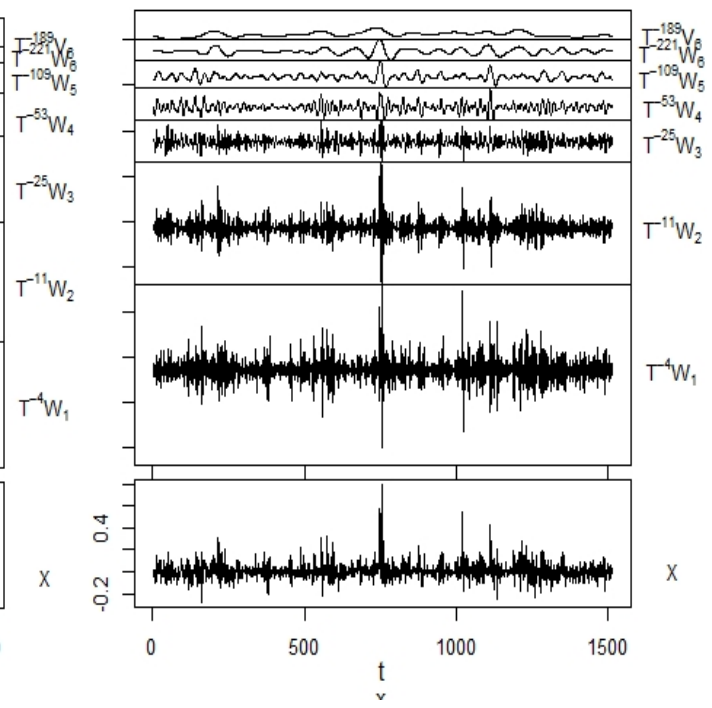

(b)

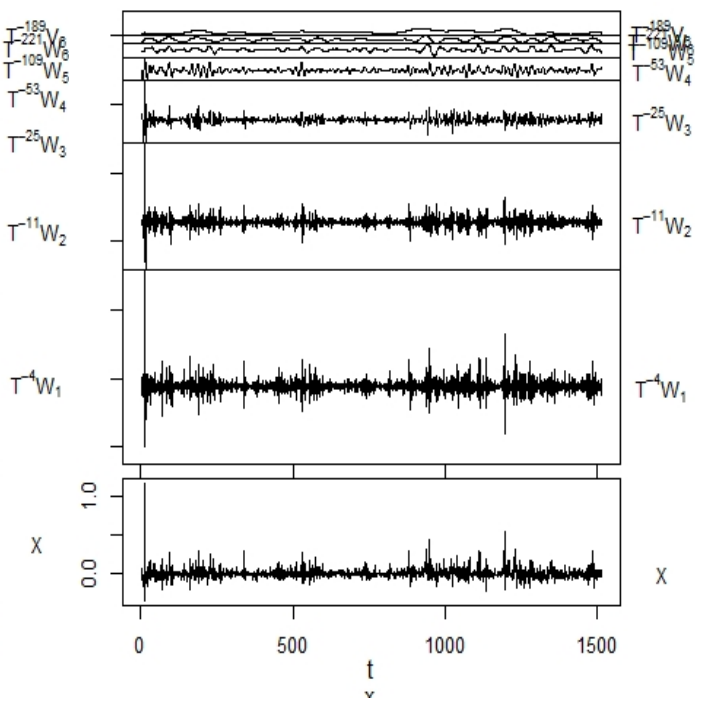

(d) 
Figure 1 Cryptocurrencies time series multi scale decomposition plot for the daily returns,

(a) Stellar (b) Dash (c) Monero (d) XBP (e) BitCoin (continued)

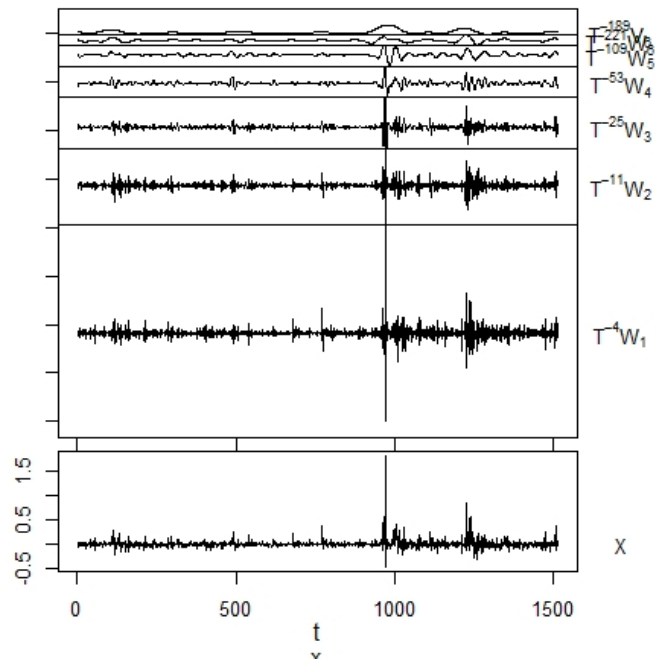

(e)

Most of the studies done on financial markets or financial assets uses time series data. Generally time series data do not follow normal distribution at the end part or tail part of the distributions as result of which it is concern for analysts (see Moinak, 2019). Fat tail financial data contains several information and it is not captured by the normal regressions and need advanced techniques. The main problem of such techniques are they consider either time or frequency domain separately to derive the study conclusions. In both the domain information contents are different as a result of which there are chances that analysts may lead to wrong decision while making investment decisions. Today researchers try to address several complex issues that are interdisciplinary in nature and need complex modelling. For example researchers now are using methods of physics to address the issues in economics and finance. One among them is Fourier transform to address the frequency component of economic and financial series data analysis. Thereafter Fourier transformation was extensible used in the economic and finance research to uncover several hidden information in the data. Similarly then wavelet methodology was introduced to analyse economic and financial data. The main advantage of using wavelet analysis is as follows. First one can study economic and finance series in both time and frequency domain together at a time. Secondly wavelet analysis is a model free approach and hence the chances of errors in analysis are negligible. Using wavelet analysis discrete data converted into the continuous series which out altering the content of the series as result one can compare both discrete and continuous data while analysing economic and finance series. Application of wavelet techniques in economic and finance research is relatively new. To study the economic and financial series, traditionally researchers used to decompose the data into daily, weekly, monthly or yearly frequency or periods. Wavelet techniques decomposes the data in to different scales in both time and frequency domain without losing any data points. At the first level of decomposition wavelet techniques decomposes into two parts generally termed as father wavelets and mother wavelets. Father wavelets represent the low frequency component of the 
economic or financial series whereas mother wavelets represent the high frequency component of the economic or financial series. To be more precise father wavelets characterises the trend component of the economic or financial series and mother wavelets characterises all the variations resulting from the trends. Resulting wavelet decomposition at different levels is shown in Figure 1.

\subsection{Wavelet coherence}

Study uses bivariate wavelet coherence and it can be defined as the wavelet correlation coefficient calculation based on the continuous wavelet transformation as shown in below equation (1).

$$
\mathbf{R}_{n}^{2}(\mathbf{s})=\frac{\left|\mathbf{S}\left(\mathbf{s}^{-1} \mathbf{W}_{\mathrm{n}}^{\mathrm{XY}}(\mathrm{s})\right)\right|^{2}}{\mathbf{S}\left(\mathbf{s}^{-1}\left|\mathbf{W}_{\mathrm{n}}^{\mathrm{X}}(\mathbf{s})\right|^{2}\right) * \mathbf{S}\left(\mathbf{s}^{-1}\left|\mathbf{W}_{\mathrm{n}}^{\mathrm{Y}}(\mathrm{s})\right|^{2}\right)}
$$

where

- $\quad \mathbf{S}$ is a smoothing operator, in time and frequency

- $\quad \mathbf{W}^{\mathbf{X}}, \mathbf{W}^{\mathbf{Y}}$ are the wavelets for each time series

- $\mathbf{W}^{\mathbf{X Y}}$ is the cross wavelet

- $\quad$ similar to a correlation coefficient varying in time and frequency

- $\quad$ get $95 \%$ confidence level using chi-squared distributions

- $\mathbf{s}^{-\mathbf{1}}$ is used to convert to energy density ( $\mathbf{s}=$ scale, $\mathbf{n}=$ time index $)$.

Wavelet coherence diagram contains phase arrows and direction of the arrows represents phase or anti-phase movements of the two time series. Phase difference is calculated by using the below equation (2).

$$
\phi_{\mathbf{n}}(s)=\tan ^{-1} \frac{\operatorname{imag}\left(S\left(s^{-1} W_{\mathbf{n}}^{X Y}(s)\right)\right)}{\operatorname{real}\left(S\left(s^{-1} W_{n}^{X Y}(s)\right)\right)}
$$

Phase arrows pointing towards:

- $\quad$ Right: in-phase

- Left: anti-phase

- Down: $\mathbf{X}$ leading $\mathbf{Y}$ by $90^{\circ}$

- Up: $\mathbf{Y}$ leading $\mathbf{X}$ by $90^{\circ}$.

The wavelet coherence plot between the cryptocurrency pairs (co-movement between the pairs) both in frequency and time domain in a single frame is shown in Figure 3. Wavelet coherence diagram is very useful to locate the time localised similar pattern if there any exists between the two time series. Common behaviour between the two time series mainly exists due to the two reasons: first when one time series is influencing the other time series and Second could be the unknown phenomenon due to which both the time series could get influenced. Wavelet coherence diagram is very useful to study the time 
series data that are non-stationary. Any phase change [as shown in equation (2)] in the wavelet coherence diagram could be interpreted as the lead or lag between the two time series. Phase difference between the two time series can be interpreted by studying the direction of the arrows in the wavelet coherence diagram. When the direction of the arrow is rightward oriented then the two time series are said to be in the phase whereas if the direction of the arrow is leftward oriented then the two time series are said to be in anti-phase. Further if the arrow head is downward oriented then time series 1 is leading time series 2 by right angle (90 degrees) whereas if the arrow head is upward oriented then time series 2 is leading time series 1 by right angle (90 degrees). White dashed line in the wavelet coherence diagram represents the cone of influence or the area where the time series coherence is significant. In Figure 3 horizontal axis shows the time horizon or all data points from 6th August 2014 to 28th September 2018 (daily data) and in the horizontal axis represents the different scale decomposition the time series in (1-2) days, (2-4) days, (4-8) days, (8-16) days, (16-32) days and more in the power of 2. Scale with different colours represents the intensity of the coherence between 0 and 1 . More the value in the scale more will be the coherence between the two time series. Warmer colours in shades of red have higher values whereas colder colours in shades of blues have lower values.

\subsection{Wavelet clustering}

Wavelet clustering is done using the Ward's minimum variance criterion to derive the conclusions. The main characteristics of the Ward method are that it uses minimum variance criteria to calculate the squared Euclidian distances between the two objects. The initial cluster squared Euclidian distance can be represented by the following equation (3).

$$
D_{i j}=d\left(\left\{X_{i}\right\},\left\{X_{j}\right\}\right)=\left\|X_{i}-X_{j}\right\|^{2}
$$

Then using the Lance-Williams algorithm, Ward's minimum variance criterion is estimated recursively using the squared Euclidian distances between the clusters.

Let assume that the next clusters to merge are $C_{i}$ and $C_{j}$, and $D_{i j}, D_{i k}, D_{j k}$ are the calculated squared Euclidian distances between the clusters $C_{i}, C_{j}$ and $C_{k}$. Then $D_{(i j) k}$ represents the squared Euclidian distances between the clusters $C_{i} U C_{j}$ and $C_{k}$. Then in that case the distance $D_{(i j) k}$ between the clusters $C_{i} U C_{j}$ and $C_{k}$ can be calculated using the Lance-Williams algorithm is as shown in equation (4).

$$
D_{(i j) k}=\alpha_{i} D_{i k}+\alpha_{j} D_{j k}+\beta D_{i j}+\gamma\left|D_{i k}-D_{j k}\right|
$$

Then using the equation (4), Ward's minimum variance criterion can be implemented for the disjoint clusters $C_{i}, C_{j}$ and $C_{k}$ with sizes $n_{i}, n_{j}$ and $n_{k}$ as shown in equation (5) below.

$$
\begin{aligned}
D_{(i j) k} & =\left\{\left(n_{i}+n_{j}\right) /\left(n_{i}+n_{j}+n_{k}\right)\right\} * D_{i k}+\left\{\left(n_{j}+n_{k}\right) /\left(n_{i}+n_{j}+n_{k}\right)\right\} \\
& * D_{j k}-\left\{\left(n_{k}\right) /\left(n_{i}+n_{j}+n_{k}\right)\right\} * D_{i j}
\end{aligned}
$$

where $\alpha_{i}, \alpha_{j}, \beta$ and $\gamma$ are the parameters

$$
\alpha_{i}=\left(n_{i}+n_{j}\right) /\left(n_{i}+n_{j}+n_{k}\right)
$$




$$
\begin{aligned}
& \beta=-\left(n_{k}\right) /\left(n_{i}+n_{j}+n_{k}\right) \\
& \gamma=0
\end{aligned}
$$

\section{Results and discussion}

Table 1 shows the descriptive statistics for all the five cryptocurrencies and Table 2 shows the co-relation among the five cryptocurrencies.

Table 1 Descriptive statistics

\begin{tabular}{lccccc}
\hline & Stellar & Dash & Monero & XBP & BitCoin \\
\hline Mean & 0.007 & 0.005 & 0.005 & 0.006 & 0.002 \\
Standard error & 0.002 & 0.002 & 0.002 & 0.002 & 0.001 \\
Median & -0.003 & -0.002 & -0.001 & -0.003 & 0.002 \\
Standard deviation & 0.092 & 0.071 & 0.074 & 0.085 & 0.039 \\
Sample variance & 0.009 & 0.005 & 0.005 & 0.007 & 0.002 \\
Kurtosis & 33.434 & 49.748 & 13.937 & 141.836 & 5.618 \\
Skewness & 3.852 & 3.675 & 1.836 & 7.939 & 0.085 \\
Range & 1.368 & 1.503 & 1.072 & 2.254 & 0.464 \\
Minimum & -0.307 & -0.347 & -0.278 & -0.460 & -0.211 \\
Maximum & 1.061 & 1.156 & 0.794 & 1.794 & 0.252 \\
Sum & 10.141 & 6.999 & 7.901 & 8.921 & 3.582 \\
Count & $1,515.000$ & $1,515.000$ & $1,515.000$ & $1,515.000$ & $1,515.000$ \\
Largest (1) & 1.061 & 1.156 & 0.794 & 1.794 & 0.252 \\
Smallest (1) & -0.307 & -0.347 & -0.278 & -0.460 & -0.211 \\
\hline
\end{tabular}

Table 2 Correlation matrix

\begin{tabular}{lccccc}
\hline & Stellar & Dash & Monero & XBP & BitCoin \\
\hline Stellar & 1 & & & & \\
Dash & 0.196 & 1 & & & \\
Monero & 0.254 & 0.357 & 1 & & \\
XBP & 0.480 & 0.133 & 0.184 & 1 & \\
BitCoin & 0.282 & 0.398 & 0.447 & 0.228 & 1 \\
\hline
\end{tabular}

Mean daily return for BitCoin (0.002) is lower as compared to the other cryptocurrencies. Similarly Stellar (0.007) has higher mean daily return as compared to the other cryptocurrencies. Stellar and XBP pair shows highest correlations $(0.480)$ whereas Dash and XBP pair shows lowest correlations (0.133) among all cryptocurrency pairs. Overall the correlations among the cryptocurrencies are within the acceptable limits (less than 0.8). Standard errors are lower among all the cryptocurrencies. Standard deviation for BitCoin (0.039) is lower as compared to the other cryptocurrencies. Similarly Stellar (0.092) has higher standard deviation as compared to the other cryptocurrencies. For all cryptocurrencies kurtosis is high that implies they have flatter tails and higher information content for making investment decisions. For all cryptocurrencies skewness are positive and expect BitCoin the values are above 1. Figure 2 shows the time series 
returns plot for daily data of all cryptocurrencies. Table 1 and Figure 1 shows BitCoin is more stable as compared to the other cryptocurrencies whereas XBP and Stellar are more volatile.

Figure 2 Cryptocurrencies time series plot for the daily returns (see online version for colours)

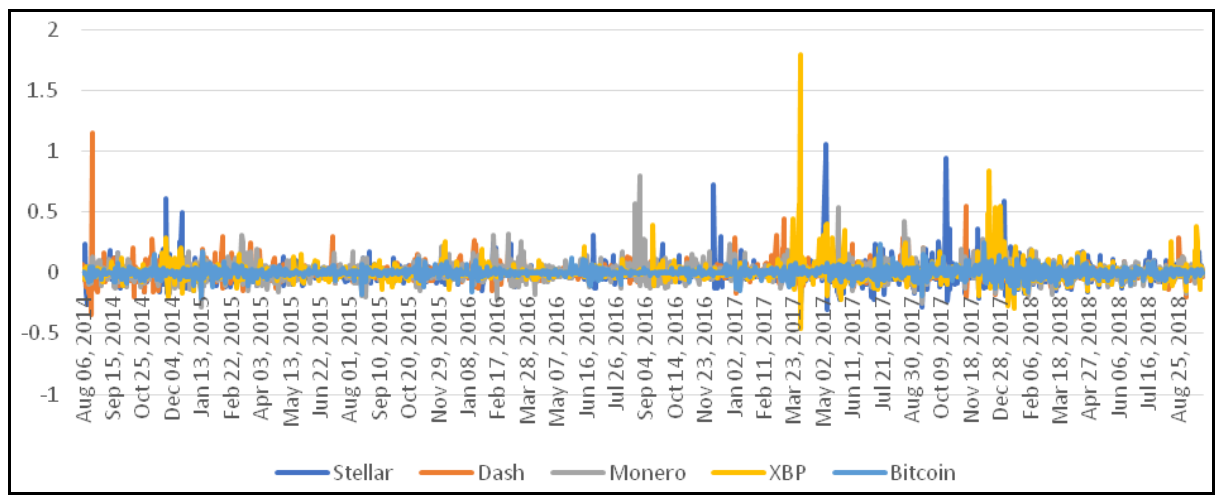

Figure 2 shows cryptocurrencies time series multi scale decomposition plot for the daily returns that enables to compare the different scales (1-2) days, (2-4) days, (4-8) days, (8-16) days, (16-32) days and more in a single frame. It is clear from the wavelet multi scale decomposition that the wavelet shape is different at every level of decompositions and the shape of the wavelet reflects the features of the data. Change in the shape of the wavelet at different levels indicates that the information content at different level are different and same could be useful for making investment decision.

Figure 3 shows the wavelet coherence plot between the cryptocurrency pairs (co-movement between the pairs) both in frequency and time domain in a single frame. Wavelet coherence diagram is very useful to locate the time localised similar pattern exists between the two time series. There could exists common behaviour between the two time series mainly due to the two reasons: first when one time series is influencing the other time series and Second could be the unknown phenomenon due to which both the time series could get influenced. Wavelet coherence diagram is very useful to study the time series data that are non-stationary. Any phase change in the wavelet coherence diagram could be interpreted as the lead or lag between the two time series. Phase difference between the two time series can be interpreted by the direction of the arrows in the wavelet coherence diagram. When the direction of the arrow is right oriented then the two time series are in phase whereas if the direction of the arrow is left oriented then the two time series are anti-phase. Further if the arrow is downward oriented then time series 1 is leading time series 2 by right angle (90 degrees) whereas if the arrow is upward oriented then time series 2 is leading time series 1 by right angle (90 degrees). White dashed line in the wavelet coherence diagram represents the cone of influence or the area where the time series coherence is significant. In Figure 3 horizontal axis shows the time horizon or all data points from 6th August 2014 to 28th September 2018 and in the horizontal axis represents the different scale decomposition the time series in (1-2) days, (2-4) days, (4-8) days, (8-16) days, (16-32) days and more. Scale with different colours represents the intensity of the coherence between 0 and 1 . More the value in the scale more will be the coherence between the two time series. Warmer colours in shades of red have higher values whereas colder colours in shades of blues have lower values. 
Figure 3 Wavelet coherence plot between the cryptocurrency pairs (co-movement between the pairs), (a) Stellar with Dash, Monero, XBP and BitCoin (b) Dash with Monero, XBP and BitCoin (c) Monero with XBP and BitCoin and XBP with BitCoin (see online version for colours)

Wavelet Coherence: A vs B

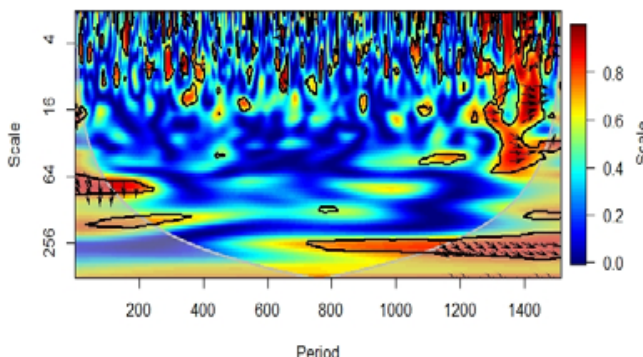

Wavelet Coherence: A vs D

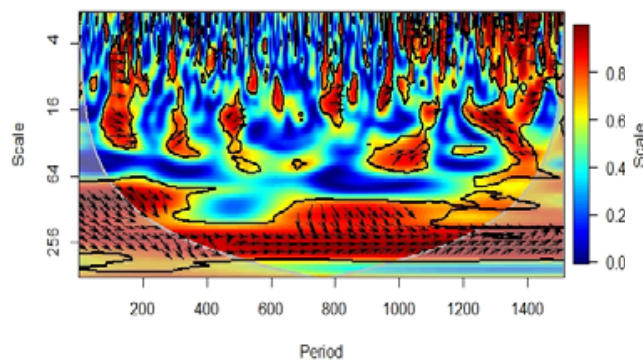

(a)

Wavelet Coherence: B vs C

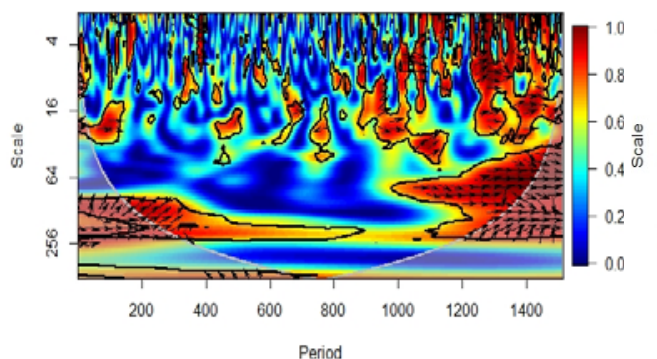

Wavelet Coherence: A vs C

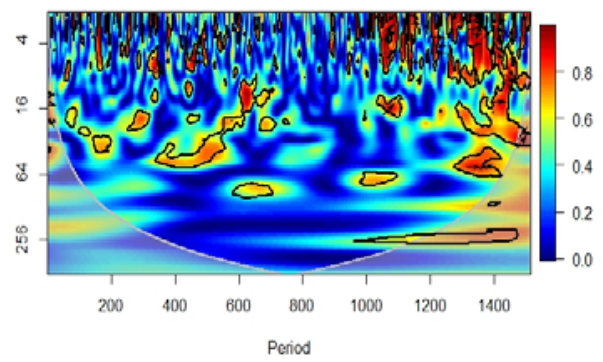

Wavelet Coherence: A vs E

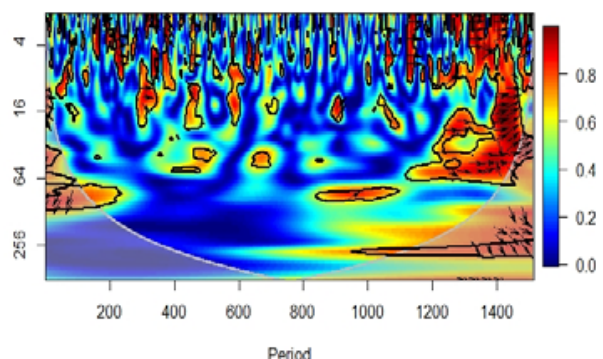

Period

Wavelet Coherence: B vs D

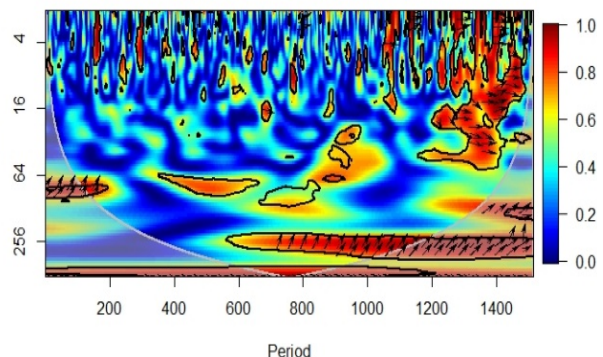

Period

Wavelet Coherence: B vs E

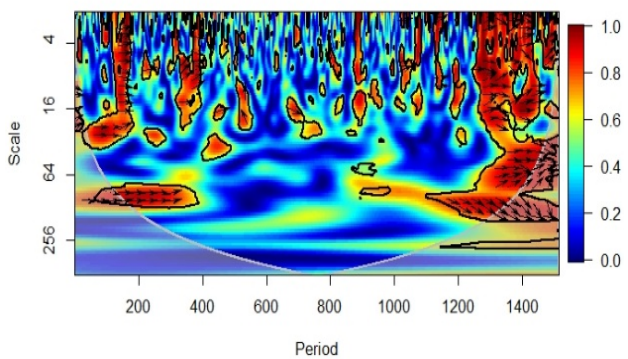

(b) 
Figure 3 Wavelet coherence plot between the cryptocurrency pairs (co-movement between the pairs), (a) Stellar with Dash, Monero, XBP and BitCoin (b) Dash with Monero, XBP and BitCoin (c) Monero with XBP and BitCoin and XBP with BitCoin (continued) (see online version for colours)

Wavelet Coherence: $\mathbf{C}$ vs $\mathbf{E}$ Wavelet Coherence: D vs E
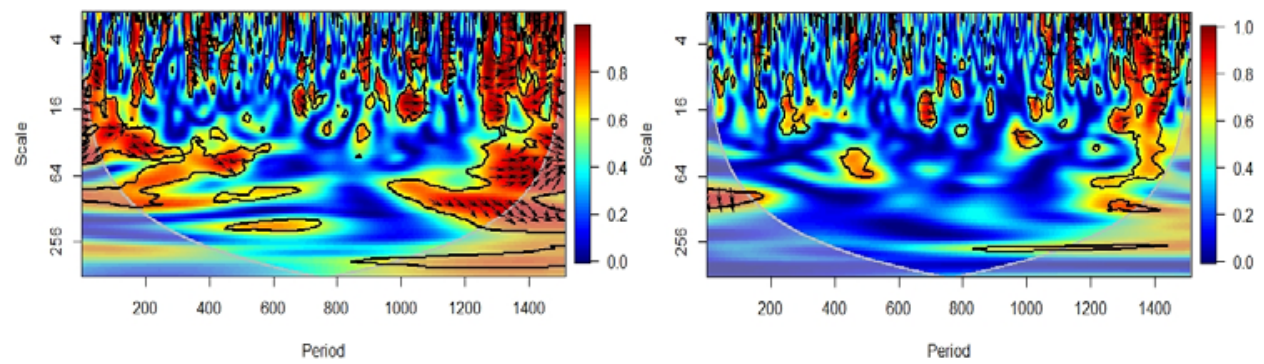

Wavelet Coherence: C vs D

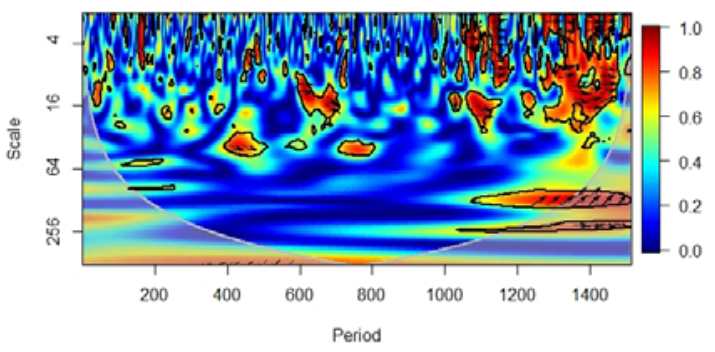

(c)

\subsection{Wavelet coherence}

- Stellar and Dash pair: For time scales (1-2) days, (2-4) days, (4-8) days are coherent in many occasions but for very small period of days and for higher scales they are not coherent. Except for few weeks in the last quarter of 2014 at scale (64-128) days and from 2016 onwards at scale (256-512) days Stellar and Dash pair is coherent. From the second quarter of 2017 Stellar and Dash pair is highly coherent for time scales (1-2) days, (2-4) days, (4-8) days, (8-16) days, (16-32) days and (32-64) days, and rightwards arrow direction shows that they are in phase.

- Stellar and Monero pair: Similar kind of observations seen in Stellar and Monero pair like Stellar and Dash pair. For time scales (1-2) days, (2-4) days, (4-8) days are coherent in many occasions but for very small period of days and for higher scales they are not coherent. From November 2016 Stellar and Monero pair is highly coherent for time scales (1-2) days, (2-4) days, (4-8) days, (8-16) days. Rightward arrow direction shows that they are in phase.

- Stellar and XBP pair: Stellar and XBP pair is coherent for time scales (1-2) days, (2-4) days, (4-8) days are coherent in many occasions but for very small period of days like previous cases but Stellar and XBP pair is highly coherent for (64-128) days, (128-256) days, (256-512) days that last for the whole period of the study sample. Rightward arrow direction shows that they are in phase. Further in most of 
the cases the arrow head are pointing downwards that implies stellar is leading over XBP.

- Stellar and BitCoin pair: Stellar and BitCoin pair wavelet coherence diagram is quite similar to the 'Stellar and Dash' and 'Stellar and Monero' pairs wavelet coherence diagram. For time scales (1-2) days, (2-4) days, (4-8) days are coherent in many occasions but for very small period of days and for higher scales they are not coherent. From 2016 Stellar and Dash pair is highly coherent for time scales (1-2) days, (2-4) days, (4-8) days, (8-16) days, (16-32) days and (32-64) days, and rightward arrow direction shows that they are in phase.

- Dash and Monero pair: Dash and Monero pair is coherent for time scales (1-2) days, (2-4) days, (4-8) days are coherent in many occasions but for very small period of days, but Dash and Monero pair is highly coherent for (64-128) days, (128-256) days, (256-512) days that last for the almost whole period of the study sample. Rightward arrow direction shows that they are in phase. Dash and Monero pair wavelet coherence diagram is quite similar to the Stellar and Monero wavelet coherence diagram.

- Dash and XBP pair: Dash and XBP pair is coherent for time scales (1-2) days, (2-4) days, (4-8) days are coherent in many occasions but for very small period of days like previous cases but Dash and XBP pair is highly coherent for (256-512) days that last for the whole period from last quarter of 2015. Rightward arrow direction shows that they are in phase. Further in most of the cases the arrow head are pointing upwards that implies XBP is leading over Dash.

- Dash and BitCoin pair: Dash and BitCoin pair is coherent for time scales (1-2) days, (2-4) days, (4-8) days are coherent in many occasions but for very small period of days like previous cases but Dash and BitCoin pair is highly coherent for time scales from (1-2) days to (128-256) days from second quarter of 2017. Rightward arrow direction shows that they are in phase.

- Monero and XBP pair: Monero and XBP pair is coherent for time scales (1-2) days, (2-4) days, (4-8) days are coherent in many occasions but for very small period of days like previous cases but Monero and XBP pair is highly coherent for (1-2) days, (2-4) days, (4-8) days, (8-16) days, (128-256) days that last for the whole period from third quarter of 2017. Rightward arrow direction shows that they are in phase.

- Monero and BitCoin pair: Monero and BitCoin pair is coherent for time scales (1-2) days, (2-4) days, (4-8) days are coherent in many occasions but for very small period of days like previous cases but Monero and BitCoin pair is highly coherent for (1-2) days, (2-4) days, (4-8) days, (8-16) days, (16-32) days, (32-64) days, (64-128) days, (128-256) days that last for the whole period from third quarter of 2017. Rightward arrow direction shows that they are in phase.

- $\quad$ XBP and BitCoin pair: XBP and BitCoin pair not much coherent for all time scales except for the period from third quarter of 2017 where it is highly coherent for time scales (1-2) days to (32-64) days. 
- Overall: Cryptocurrency pairs are coherent for very shorter period of time especially at time scales (1-2) days, (2-4) days, (4-8) days. Higher coherence observed for almost all pairs from last quarter of 2017 for scales (1-2) days to (32-64) days. Overall wavelet coherence diagrams clearly indicate about the contagion effect among the cryptocurrency pairs for this period. Finally rightward arrow direction shows that they are in phase.

\subsection{Wavelet clustering}

Figure 4 shows wavelet clustering diagram for cryptocurrencies. Horizontal axis represents different cryptocurrencies and vertical axis represents dissimilarities. Dissimilarity scores for XBP and BitCoin are the lowest among the cryptocurrencies. Investors those who are willing to invest only in XBP and BitCoin cryptocurrencies is not going to get any benefit from diversification. Further inclusion of Dash in the two cryptocurrencies group 'XBP and BitCoin' overall dissimilarity scores increased. Stellar is the next to join the three cryptocurrencies group 'XBP, BitCoin and Dash', inclusion of stellar further increases the value of dissimilarly. Finally higher amount of dissimilarity can be achieved by adding Monero to the group of four cryptocurrencies group 'XBP, BitCoin, Dash and Stellar'. From the investment point of view in cryptocurrencies: let assume investor 1 invested in all five cryptocurrencies and get maximum benefit from the portfolio diversifications. Also assume that there is an another investor 2, who is also investing in cryptocurrencies and only in the XBP and BitCoin cryptocurrencies. Then in such case according to the portfolio diversification theory, investor 2 has a risky investment as both the cryptocurrencies looks identical and will not get any benefits of diversifications from these investment. However investor 2 can reduces it total investment risk by additionally investing in the Dash cryptocurrency and so on. The wavelet clustering diagram states that every time investor 2 additionally invests in the new cryptocurrency realise the benefit of portfolio diversification and once the investment is transmitted to the all five cryptocurrencies, investor 2 will realise the benefit of portfolio diversifications as similar to the investor 1 .

Figure 4 Wavelet clustering of cryptocurrencies

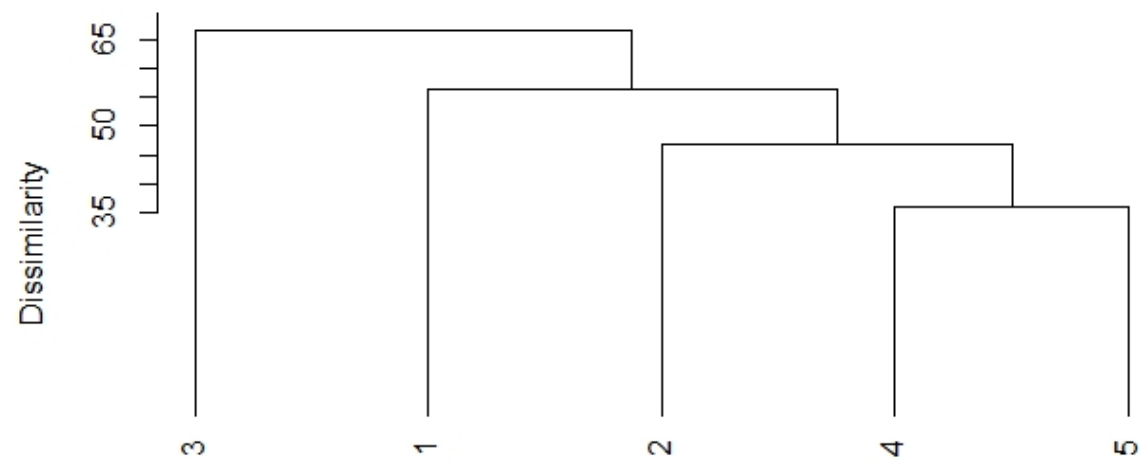

Notes: 1 - Stellar; 2 - Dash; 3 - Monero; 4 - XBP; 5 - BitCoin. 


\section{Conclusions}

Significant development and growth in the cryptocurrency market opens up a new avenue for investments and business. In the recent past cryptocurrencies not only total market capitalisation jumps up but at the same time volume of cryptocurrencies traded also increases. Risk return trade off for the cryptocurrencies are different from that of the stocks, commodities and other assets. In past several studies address the co-movements of cryptocurrencies with other assets but none of the studies addressed whether there is a co-movement among the cryptocurrencies. The present study highlighted the same, in addition to it several interesting facts related to cryptocurrencies co-movements both in time and frequency domain are highlighted by the present study. The present study focuses on five cryptocurrencies co-movements physiognomies both in time and frequency domain. The study begins with investigating the correlations among the five cryptocurrencies selected based on their higher market capitalisation and long time period. Study finds that Stellar and XBP pair shows highest correlations (0.480) whereas Dash and XBP pair shows lowest correlations (0.133) among all cryptocurrency pairs. Mean daily return for BitCoin (0.002) is lower and higher for Stellar (0.007) as compared to the other cryptocurrencies. Stellar and XBP pair shows highest correlations $(0.480)$ whereas Dash and XBP pair shows lowest correlations (0.133) among all cryptocurrency pairs. Standard errors are lower among all the cryptocurrencies. Standard deviation for BitCoin (0.039) is lower as compared to the other cryptocurrencies. Similarly Stellar (0.092) has higher standard deviation as compared to the other cryptocurrencies. For all cryptocurrencies kurtosis is high that implies they have flatter tails and higher information content for making investment decisions. For all cryptocurrencies skewness are positive and expect BitCoin the values are above 1. From daily time series return plot pattern of cryptocurrencies study finds that BitCoin is more stable as compared to the other cryptocurrencies whereas XBP and Stellar are more volatile. Then wavelet coherence diagrams indicate in general that all cryptocurrency pairs are coherent for very shorter period of few days for several times or discrete number of times especially at time scales (1-2) days, (2-4) days, (4-8) days. Higher continuous coherence observed for almost all pairs from last quarter of 2017 for scales (1-2) days to (32-64) days. Overall wavelet coherence diagrams clearly indicate about the very short and long contagion effect among the cryptocurrency pairs for the whole study period. The contagion effect is different at different time scales. Finally, rightward arrow direction shows that they are in phase. Study finds that especially Stellar and XBP pair is highly coherent for (64-128) days, (128-256) days, (256-512) days that last for the whole period of the study sample. Wavelet clustering diagram shows that Monero has highest dissimilar values among the cryptocurrencies. Whereas XBP and BitCoin has lowest dissimilar values among the cryptocurrencies. Then, wavelet clustering diagram indicates that by investing only in XBP and BitCoin cryptocurrencies investors are not going to get any benefit from diversification. Then study discussed about the investment strategy by which investors considering investment in cryptocurrencies in their investment portfolio can take full advantage of portfolio diversifications by reducing their total investment risk. This predictable co-movements pattern could be the basic investment strategies to gain maximum profit by diversifying the risk in cryptocurrency investments. 


\section{References}

Bariviera, A., Basgall, M., Hasperue, W. and Naiouf, M. (2017) 'Some stylized facts of the Bitcoin market', Physica A, Vol. 484, pp.82-90.

Bhuiyan, R.A., Rahman, M.P., Saiti, B. and Ghani, G.M. (2018) 'Financial integration between sukuk and bond indices of emerging markets: insights from wavelet coherence and multivariate-GARCH analysis', Borsa Istanbul Review, Vol. 18, No. 3, pp.218-230.

Blau, B. (2017) 'Price dynamics and speculative trading in Bitcoin', Research in International Business and Finance, Vol. 41, pp.493-499.

Bouri, E., Gupta, R., Tiwari, A.K. and Roubaud, D. (2017a) 'Does Bitcoin hedge global uncertainty? Evidence from wavelet-based quantile-in-quantile regressions', Finance Research Letters, Vol. 23, pp.87-95.

Bouri, E., MolnÃąr, P., Azzi, G., Roubaud, D. and Hagfors, L. (2017b) 'On the hedge and safe haven properties of Bitcoin: Is it really more than a diversifier?', Finance Research Letters, Vol. 20, pp.192-198.

Caporale, G.M. and Plastun, O. (2017) The Day of the Week Effect in the Crypto Currency Market (October 20, 2017), Working Paper Nos. 17-19, Department of Economics and Finance, Brunel University London [online] https://ssrn.com/abstract=3056229.

Caporale, G.M., Gil-Alana, L. and Plastun, A. (2018) 'Persistence in the cryptocurrency market', Research in International Business and Finance.

Chaudhuri, K. (1997) 'Cointegration, error correction and granger causality: an application with Latin American stock markets', Applied Economics Letters, Vol. 4, No. 8, pp.469-471.

Cheah, E-T. and Fry, J. (2015) 'Speculative bubbles in Bitcoin markets? An empirical investigation into the fundamental value of Bitcoin', Economics Letters, Vol. 130, pp.32-36.

Corbet, S., Meegan, A., Larkin, C., Lucey, B. and Yarovaya, L. (2018) 'Exploring the dynamic relationships between cryptocurrencies and other financial assets', Economics Letters, Vol. 165, pp.28-34.

European Banking Authority (2014) Opinion on 'Virtual Currencies' [online] https://eba.europa.eu/documents/10180/657547/EBA-Op-201408+Opinion+on+Virtual+Currencies.pdf (accessed 1 February 2019).

European Central Bank (2012) Virtual Currency Scheme, October [online] https://www.ecb.europa.eu/pub/pdf/other/virtualcurrencyschemes201210en.pdf (accessed 1 February 2019).

Financial Action Task Force (2014) Virtual Currencies: Key Definitions and Potential AML/CFT Risks.

Forbes, K. and Rigobon, R. (2002) 'No contagion, only interdependence: measuring stock market comovements', Journal of Finance, Vol. 57, No. 5, pp.2223-2261.

Granger, C. and Morgenstern, O. (1970) The Predictability of Stock Market Prices, Heath Lexington Books, Heath \& Co, Lexington, KY.

Ji, Q., Bouri, E., Lau, C.K.M. and Roubaud, D. (2018) 'Dynamic connectedness and integration in cryptocurrency markets', International Review of Financial Analysis.

Katsiampa, P. (2017) 'Volatility estimation for Bitcoin: a comparison of GARCH models', Economics Letters, Vol. 158, pp.3-6.

Kurihara, Y. and Fukushima, A. (2017) 'The market efficiency of Bitcoin: a weekly anomaly perspective', J. Appl. Finance Bank, Vol. 7, No. 3, pp.57-64.

Liu, Y. and Tsyvinski, A. (2018) Risks and Returns of Cryptocurrency, No. w24877, National Bureau of Economic Research.

Malkiel, B.G. and Fama, E.F. (1970) 'Efficient capital markets: a review of theory and empirical work', The Journal of Finance, Vol. 25, No. 2, pp.383-417.

Markwat, T., Kole, E. and Van Dijk, D. (2009) 'Contagion as a domino effect in global stock markets', Journal of Banking \& Finance, Vol. 33, No. 11, pp.1996-2012. 
Moinak, M. (2019) 'OLS versus quantile regression in extreme distributions', Contaduria y Administración, Vol. 64, No. 2, p.e102 [online] http://dx.doi.org/10.22201/fca.24488410e. 2018.1702 .

Patel, R.J. (2017) 'Co-movement and integration among stock markets: a study of 14 countries', Indian Journal of Finance, Vol. 11, No. 9, pp.53-66.

Pieters, G. and Vivanco, S. (2017) 'Financial regulations and price inconsistencies across Bitcoin markets', Information Economics and Policy, Vol. 39, pp.1-14.

Vandezande, N. (2017) 'Virtual currencies under EU anti-money laundering law', Computer Law and Security Review, Vol. 33, No. 3, pp.341-353.

Vyklyuk, Y., Vukovic, D. and Jovanović, A. (2013) 'Forex prediction with neural network: USD/EUR currency pair', Actual Problem of Economics, Vol. 10, No. 148/2013, pp.251-261. 